\title{
Modelling Transmission of Buruli Ulcer in the Central Region of Ghana
}

\author{
Nyarko Christiana Cynthia, Nyarko Peter Kwesi, Ampofi Isaac, Asante Emmanuel
}

Mathematical Sciences Department, University of Mine and Technology, Tarkwa, Ghana

\section{Email address:}

ccnyarko@umat.edu.gh (N. C. Cynthia)

\section{To cite this article:}

Nyarko Christiana Cynthia, Nyarko Peter Kwesi, Ampofi Isaac, Asante Emmanuel, Modelling Transmission of Buruli Ulcer in the Central Region of Ghana. Mathematical Modelling and Applications. Vol. 5, No. 4, 2020, pp. 221-231. doi: 10.11648/j.mma.20200504.13

Received: June 11, 2020; Accepted: September 10, 2020; Published: December 31, 2020

\begin{abstract}
The pathogen of Buruli Ulcer (BU) is known to be Mycobacterium Ulcerans whose mode of transmission is entirely not known, although the disease is recognised to be associated with contaminated water. The hypothesised transmission involves humans being bitten by the water bugs (vector) that prey on mollusks, snails and young fishes. The hypothesised transmission also involves humans feeding on an infected fish or frog. This study seeks to contribute to the dynamics and analyses of the transmission mechanism of Buruli Ulcer in communities along Offin River in the Central Region of Ghana. The model equilibria were determined and conditions for the equilibria were also established. The basic reproduction number, $R_{0}$ was derived using the Next Generation approach and its estimated value was 1.20771. The result reveals that, $R_{0}$ is greater than 1 , indicating a horizontal spread of the infection across the population. The transmission dynamics of Buruli Ulcer model of the Susceptible, Infected and Recovered (SIR) type also show that the disease will continue to spread at the study areas as long as the reservoir for Mycobacterium Ulcerans continue to sustain enough infected water bugs and infected fish or infected frog to contain the disease. The study further concludes that, the rate of spread of Buruli Ulcer in the affected communities continue to be high due to its mode of transmission. This study suggest that adequate control measures including mass education and prompt treatment to curb the spread should be emphasized.
\end{abstract}

Keywords: Pathogen, Buruli, Ulcer, Ulcerans, Spread, Infected, Stagnant, Humans, Vector

\section{Introduction}

Modelling of infectious disease is a tool which has been used to study the means by which diseases spread, forecast the future course of an outbreak and to evaluate strategies to control an epidemic [1]. Mathematical models may not offer comprehensive descriptions of how to control diseases. They are elegant methods for evaluating the possible influence of different strategies offered in public health intervention programs [2].

Buruli Ulcer (BU) is a disease of the skin caused by Mycobacterium Ulcerans, a slow growing mycobacterium that classically infect the skin and subcutaneous tissue, giving rise to indolent no ulcerated (nodules, plaques) and ulcerated lesions. After tuberculosis and leprosy, Buruli Ulcer is the third most common mycobacteriosis of human. The mode of Mycobacterium Ulcerans transmission is not fully understood in the study area, although the disease is recognized to be associated to contaminated water. Areas affected by Buruli Ulcer disease are located near stagnant or slow-moving water, and outbreaks appear to be related to environmental changes (deforestation, agriculture, hydraulic installations) involving surface water [3].

The occurrence of Buruli Ulcer upstream of the Offin River is greater and steeply dwindles as you go beyond Dunkwa. So, the communities on the upstream of river Offin from Agroyesum (e.g. Subin, Ameyaw, Betenase, Ampabena, Nkotumso, Dominase, Ayanfuri, Powerline, Obiarabiaradanedanmu, Pokukrom and Dunkwa) are very endemic [3]. Therefore, to provide awareness of this endemic, this paper seeks to develop a transmission model (deterministic model) of Buruli Ulcer and help to identify control measures that will minimize the disease in the study area.

\section{Literature Review}

In Africa, the history of Buruli Ulcer can be divided into two main periods: before 1980 and after 1980. There were many important literatures before 1980 on the disease in 
several African countries: Cameroon, the Democratic Republic of the Congo, Gabon, Ghana, Nigeria and Uganda. In the Central African Republic, Kenya, Sudan, and the United Republic of Tanzania, cases were suspected but never confirmed. The most significant contributions came from the Democratic Republic of the Congo and Uganda. The Uganda Buruli Group studied the clinic pathological and epidemiological aspects of the disease extensively, and opted for the term "Buruli Ulcer" because large numbers of cases were first detected in the district of Buruli near Lake Kyoga [4].

After 1980, new foci of Buruli Ulcer emerged in West Africa. A dramatic increase in the incidence of the disease is now reported in several West Africa countries, especially in Benin, Cote d'Ivoire and Ghana [5].

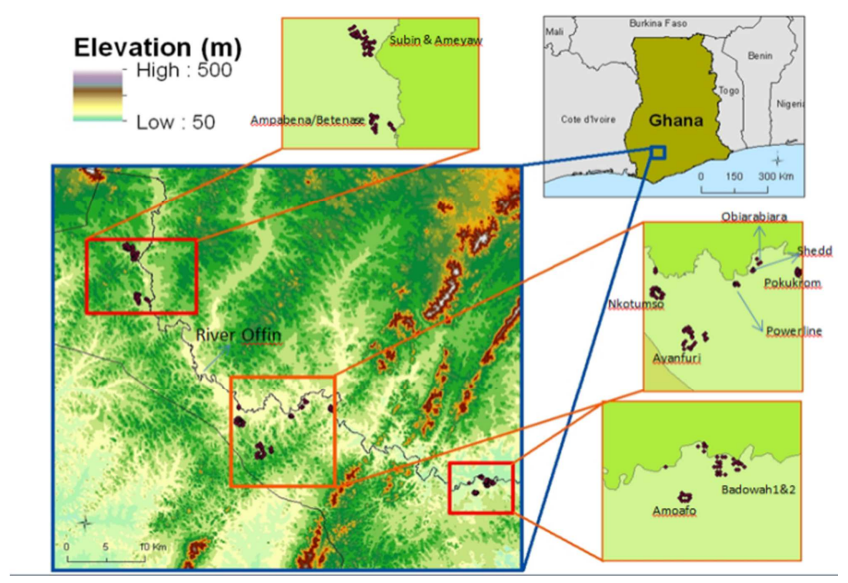

Figure 1. Study Communities (Source [3]).

The communities along Offin River in the Central Region of Ghana are endemic to Buruli Ulcer disease. Figure 1 present the communities along Offin River. These areas of study are located near stagnant or slow-moving water, and experience similar environmental changes (deforestation, agriculture, and mining) involving surface water. The primary means of securing the necessities of life in these communities are farming and mining [3]. It can be seen from the Powerline community (Figure 2), most of the cases are in Zones 3 and 4 and also at the fringes of the community. Communities such as Betenase, Ampabena (Figure 1) have Buruli Ulcer cases all over the community which seems to portray that the whole community is endemic.

Figure 3 portrays that Zone 1 of Ayamfuri has about $70 \%$ of Buruli Ulcer cases and even in that zone, 90\% were clustered together in a small sector within the centre of the town.

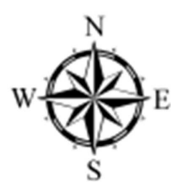

00.000200040 .0008 Decimal Degrees

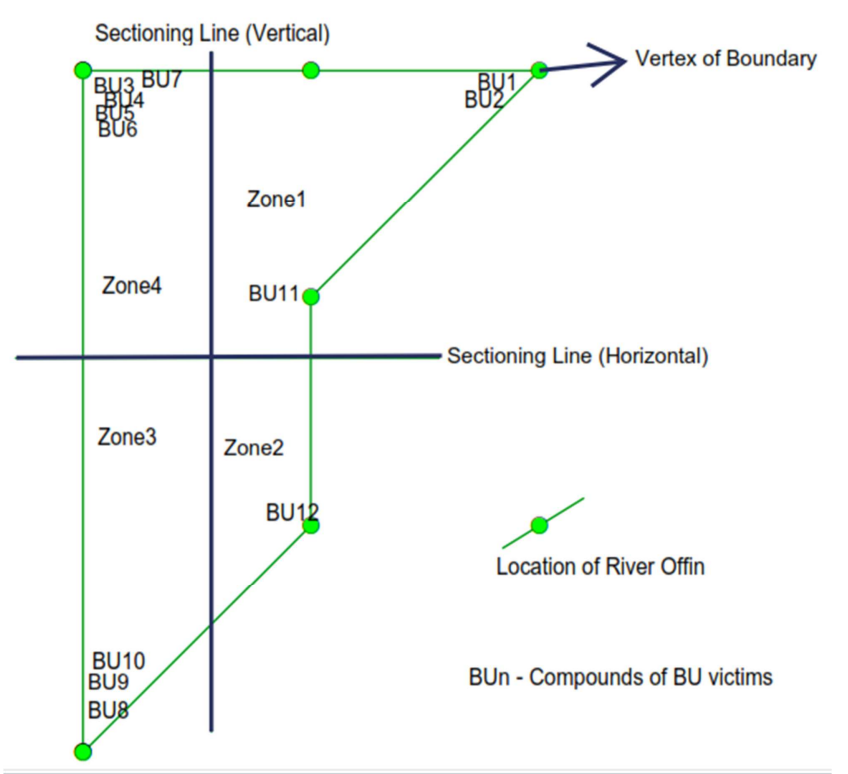

Figure 2. Map of Powerline (Source [3]).

Scot proposed a mathematical model of the SIR-type in an endeavour to explain the role of aquatic insects and arsenic in the spread of Buruli ulcer disease [6]. The paper considered arsenic environment as a reservoir for Mycobacterium ulcerans and water bug (vector) for Buruli Ulcer disease. In their model, they proposed that Buruli Ulcer is a micro parasitic disease in which host parasite interaction basically occurs within isolated communities. Again, it was assumed that the host population is of fixed size containing susceptible individuals who are not yet infected with Mycobacterium Ulcerans. It also assumed humans who develop Buruli Ulcer become immune to any further attack and this assumption led them to the SIR model. The model equations describing the proportion of humans infected by Mycobacterium Ulcerans and the corresponding proportion of water-bugs according to them are given by:

$$
\left.\begin{array}{l}
\frac{d x}{d t}=\operatorname{maby}(1-x)-r x \\
\frac{d y}{d t}=a_{1} x(1-y)-(\mu-\alpha) y
\end{array}\right\}
$$

Table 1. Description of Parameters used in the Equation (1).

\begin{tabular}{ll}
\hline Symbol & Description \\
\hline$m$ & Density of water bugs (Number of water bugs per human host) \\
$a$ & Bite frequency (biting rate of human by single water-bug) \\
$a_{1}$ & Rate of ingestion of MU by water bugs \\
$b$ & Proportion of infected bites on humans that produce infection \\
$\alpha$ & Relative concentration as in water \\
$\mu$ & Mortality rate of water-bugs \\
$x$ & Proportion of humans infected by MU \\
$y$ & Proportion of water-bugs infected by MU \\
$r$ & Death rate of humans \\
\hline
\end{tabular}




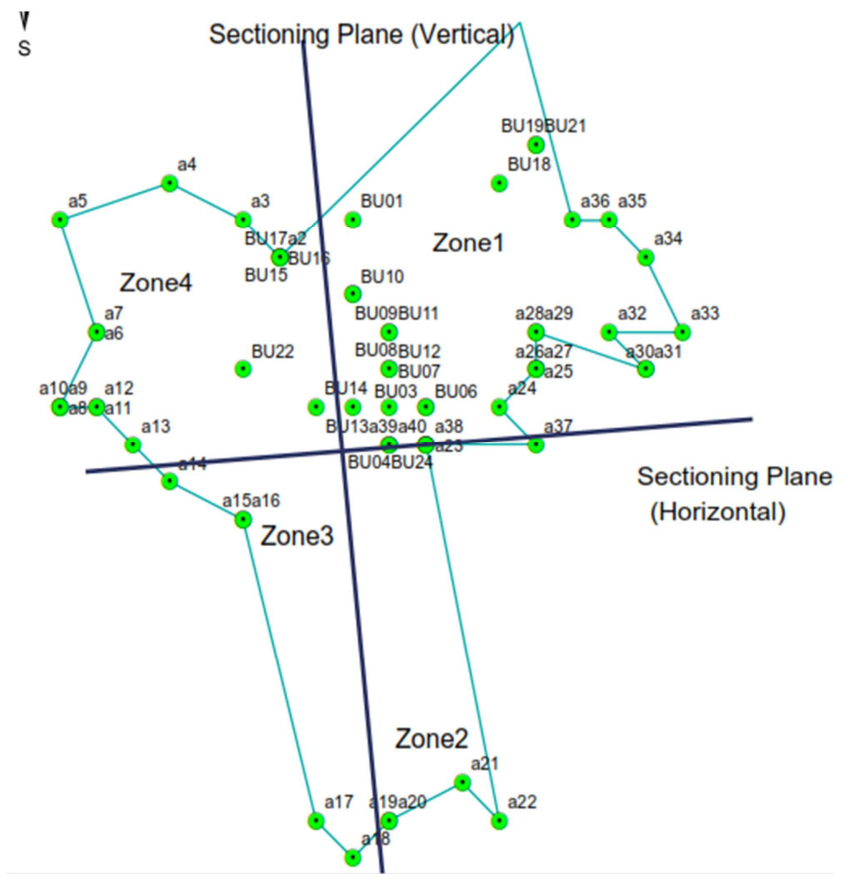

Figure 3. Map of Ayanfuri (Source [3]).

The study displayed a nonlinear relationship between the basic reproductive number $\left(R_{0}\right)$ and the widespread of infection of both infected water bugs and infected humans at a particular time. However, a small increase in the reproductive number lead to a large change in both the prevalence levels of humans and water bugs. It was deduce from their graph that, higher levels of $\left(R_{0}\right)$ will lead to increases in cases of Buruli Ulcer for $R_{0}>1$. Thus, if Buruli
Ulcer is not controlled it will continue to spread in regions with unconducive conditions. Nyabadza and Bonyah develop the transmission dynamics of Buruli Ulcer in Ghana: Insights through a mathematical model [7]. In their paper, a model for the transmission of Mycobacterium Ulcerans to human in the presence of a preventive strategy was proposed and analysed. The model equilibria were determined and conditions for the existence of the equilibria established. The model analysis was carried out in terms of the reproduction number $R_{0}$. The disease-free equilibrium was found to be locally asymptotically stable for $R_{0}<1$. The model was then fitted to data from Ghana. The dynamics of the disease was described by the following set of nonlinear set differential equations;

$$
\begin{aligned}
\frac{d S_{H}}{d t} & =\mu_{H} N_{H}+\theta R_{H}-\frac{\beta_{H} S_{H} I_{V}}{N_{H}}-\mu_{H} S_{H} \\
\frac{d I_{H}}{d t} & =\frac{\beta_{H} S_{H} I_{V}}{N_{H}}-\left(\gamma+\mu_{H}\right) I_{H} \\
\frac{d R_{H}}{d t} & =\gamma I_{H}-\left(\theta+\mu_{H}\right) R_{H} \\
\frac{d S_{V}}{d t} & =\mu_{V} N_{V}-\frac{\beta_{V} S_{V} I_{F}}{N_{V}}-\frac{\eta_{V} \beta_{V} S_{V} U}{K}-\mu_{V} S_{V} \\
\frac{d I_{V}}{d t} & =\frac{\beta_{V} S_{V} I_{F}}{N_{V}}+\frac{\eta_{V} \beta_{V} S_{V} U}{K}-\mu_{V} I_{V} \\
\frac{d S_{F}}{d t} & =\mu_{F} N_{F}-\frac{\beta_{F} I_{F} U}{K}-\frac{\eta_{F} \beta_{F} I_{F} U}{K}-\mu_{F} S_{F} \\
\frac{d I_{F}}{d t} & =\frac{\beta_{F} I_{F} U}{K}+\frac{\eta_{F} \beta_{F} I_{F} U}{K}-\mu_{F} I_{F} \\
\frac{d U_{M}}{d t} & =\sigma_{F} I_{F}+\sigma_{V} I_{V}-\mu_{E} U
\end{aligned}
$$

Table 2. Description of parameters used in Equation (2).

\begin{tabular}{ll}
\hline Symbol & Description \\
\hline$\beta_{H}$ & The effective contact rate between the vector and susceptible human \\
$\beta_{V}$ & The effective contact rate between fish and susceptible vector \\
$\beta_{F}$ & The effective contact rate between the susceptible fish and Mycobacterium Ulcerans \\
$\gamma$ & The recovery rate of infected humans \\
$\theta$ & The rate of loss of immunity of recovered humans \\
$\mu_{H}$ & Birth rate of the human population \\
$\mu_{V}$ & Birth rate of the vector population \\
$\mu_{F}$ & Birth rate of the fish population \\
$r V$ & The growth rate of the vector population \\
$r F$ & The growth rate of the fish population \\
$K$ & The environmental carrying capacity of the bacteria population \\
$\sigma_{F}$ & Rate of shedding of Mycobacterium Ulcerans into the environment by fish-frog \\
$\sigma_{V}$ & Rate of shedding of Mycobacterium Ulcerans into the environment by the vector \\
$\mu_{E}$ & Rate at which Mycobacterium Ulcerans are cleared from the environment. \\
\hline
\end{tabular}

The model exhibits a backward bifurcation and the endemic equilibrium point is globally stable when $R_{0}<1$. Sensitivity analysis also showed that the Buruli Ulcer epidemic is highly influenced by the shedding and clearance rates of Mycobacterium Ulcerans in the environment. The model was found to fit reasonably well to data from Ghana and projections on the future of the Buruli Ulcer epidemic were also made.

\section{Methodology}

A constant human population $P_{H}(t)$, the vector population of insect (e.g. water bugs, mosquitoes etc.) $P_{V}(t)$, the fishfrog population, $P_{F F}(t)$ at any time $(t)$ was considered. The 
total human population is divided into three epidemiological subclasses of those that are susceptible $S_{H}(t)$, the infected $I_{H}(t)$ and the recovered who is able to resist the infection owning to the presence of a particular treatment $R_{H}(t)$. Total vector population at any time $\mathrm{t}$ is divided into two subclasses of those that are susceptible vector $S_{V}(t)$ and those that are infectious and can transmit the Buruli ulcer to humans $I_{V}(t)$. The total population reservoir of fish-frog is also divided into two compartments of susceptible fish $S_{F F}(t)$ and infected fish $I_{F F}(t)$. Consideration of the role of the environment was made by introducing a compartment $U_{M}$, representing the density of Mycobacterium Ulcerans in the environment. Assumptions of the model is;

Mycobacterium Ulcerans are transferred from vector (water bug), fish and frog to the humans.

There is homogeneity of human, vector and fish-frog population's interactions.

Infected humans recover and are temporarily immune, but eventually lose immunity.

Fish and amphibian (frog) are preyed on by the vector and the human.

The acquiring of Mycobacterium Ulcerans through environmental contact and direct person-to-person transmission is rare [8].

Susceptible host are more likely to be infected by the disease through biting by an infectious vector (water bug). $\beta_{H}$ Being the effective biting rate that an infectious vector has to susceptible host and $\left(\beta_{H} S_{H} I_{V}\right) / P_{H}$ being the incidence of new infections transmitted by a vector. $\left(\beta_{H} S_{H} I_{F F}\right) / P_{H}$ Being the incidence of new infections transmitted to a human when the human prayed on an infected fish or frog.

Susceptible insect is infected at a rate $\left(\beta_{V} S_{V} I_{F F}\right) / P_{V}$ through the predation of infected fish or frog and $\left(\eta \beta_{V} S_{V} U_{M}\right) / K$ representing other sources in the environment. Where $\eta$ differentiates the infectivity potential of the fish-frog from that of the environment.

The vector population and the fish-frog population are assumed to be constant. Their growth functions are given by $g\left(P_{V}\right)$ and $g\left(P_{F F}\right)$ respectively. Generally, we can assume that $g\left(P_{V}\right)=\mu_{V} P_{V}$ and $g\left(P_{F F}\right)=\mu_{F} P_{F F}$.

There is a proposed hypothesis that environmental mycobacteria in the bottoms of swamps may mechanically concentrated by small water-filtering organisms such as microphagous fish, snails, mosquito larvae, small crustaceans and protozoa [9]. It can therefore be assumed that fish-frog increase the environmental concentrations of mycobacterium Ulcerans at a rate $\sigma_{F F}$. Humans are assumed not to shed any bacteria into the environment.

Vector release bacteria into the environment at a rate $\sigma_{V}$.

The model does not include a potential route of direct contact with the bacterium in water.

The birth rate of the human population is directly proportional to the size of the human population.

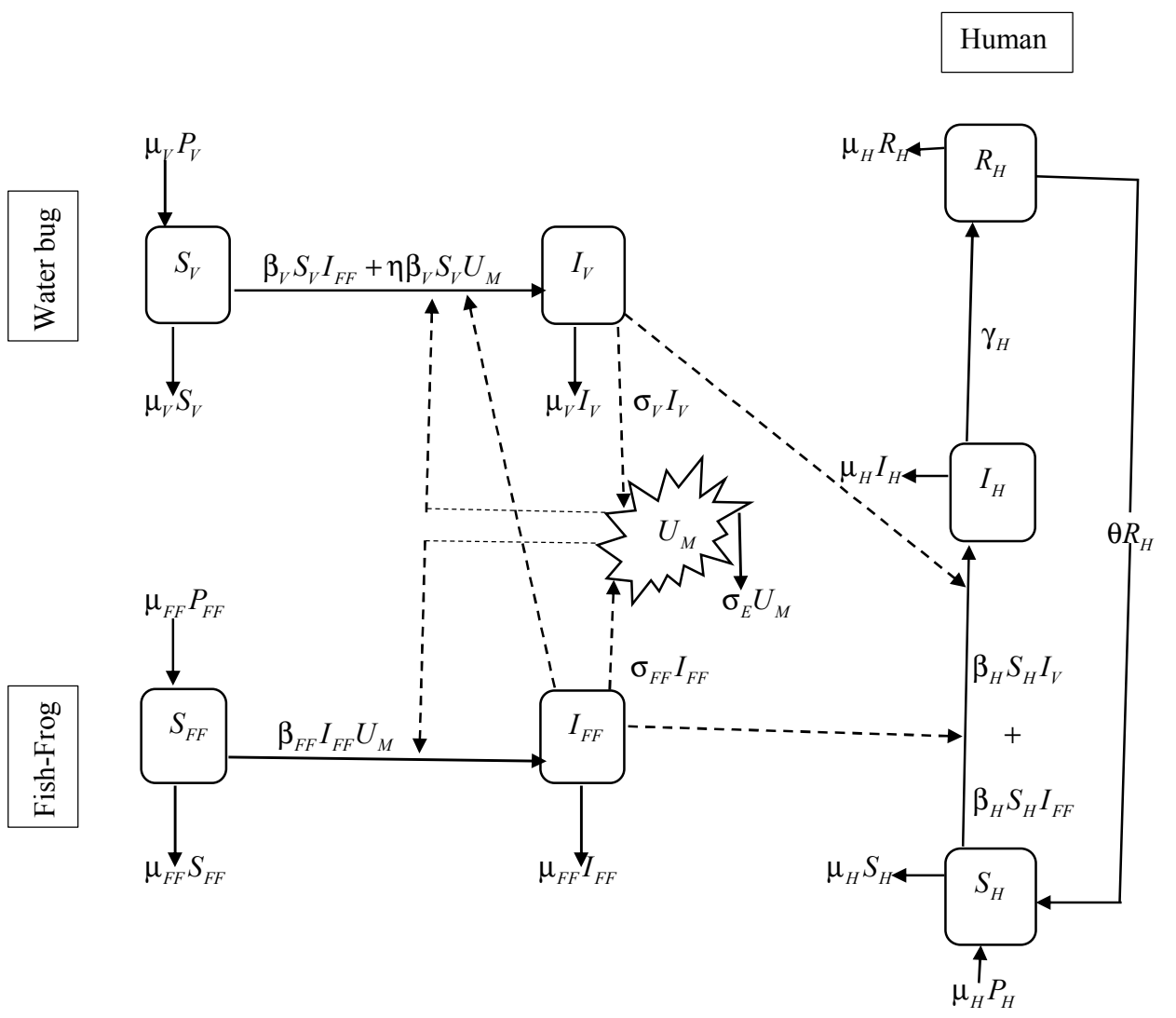

Figure 4. Transmission Dynamics of Buruli Ulcer among Fish-Frog, Water bug and Human. 
Hence the dynamics of Buruli ulcer can be described by the following set of nonlinear differential equations;

$$
\begin{aligned}
\frac{d S_{H}}{d t} & =\mu_{H} P_{H}+\theta R_{H}-\frac{\beta_{H} S_{H} I_{V}}{P_{H}}-\frac{\beta_{H} S_{H} I_{F F}}{P_{H}}-\mu_{H} S_{H} \\
\frac{d I_{H}}{d t} & =\frac{\beta_{H} S_{H} I_{V}}{P_{H}}+\frac{\beta_{H} S_{H} I_{F F}}{P_{H}}-\left(\gamma+\mu_{H}\right) I_{H} \\
\frac{d R_{H}}{d t} & =\gamma I_{H}-\left(\theta+\mu_{H}\right) R_{H} \\
\frac{d S_{V}}{d t} & =\mu_{V} P_{V}-\frac{\beta_{V} S_{V} I_{F F}}{P_{V}}-\frac{\eta \beta_{V} S_{V} U_{M}}{K}-\mu_{V} S_{V} \\
\frac{d I_{V}}{d t} & =\frac{\beta_{V} S_{V} I_{F F}}{P_{V}}+\frac{\eta \beta_{V} S_{V} U_{M}}{K}-\mu_{V} I_{V} \\
\frac{d S_{F F}}{d t} & =\mu_{F F} P_{F F}-\frac{\beta_{F F} I_{F F} U_{M}}{K}-\mu_{F F} S_{F F} \\
\frac{d I_{F F}}{d t} & =\frac{\beta_{F F} I_{F F} U_{M}}{K}-\mu_{F F} I_{F F} \\
\frac{d U_{M}}{d t} & =\sigma_{F F} I_{F F}+\sigma_{V} I_{V}-\mu_{E} U_{M}
\end{aligned}
$$

\subsection{Positivity of Solution in Equation (3)}

Let $t_{1}=\sup \left(t>0 \mid S_{H}>0, I_{H}>0, R_{H}>0, S_{V}>0\right.$, $I_{V}>0, S_{F F}>0, I_{F F}>0$ and $\left.U_{M}>0\right) \in[0, t]$

Considering the susceptible human compartment of Equation (3.6) of the model,

$$
\frac{d S_{H}}{d t} \geq \mu_{H} P_{H}+\theta R_{H}-\frac{\beta_{H} S_{H} I_{V}}{P_{H}}-\frac{\beta_{H} S_{H} I_{F F}}{P_{H}}-\mu_{H} S_{H}
$$

Factorising $S_{H}$ out of Equation (3) gives Equation (4)

$$
\frac{d S_{H}}{d t} \geq \mu_{H} P_{H}+\theta R_{H}-\left(\frac{\beta_{H} I_{V}}{P_{H}}+\frac{\beta_{H} I_{F F}}{P_{H}}+\mu_{H}\right) S_{H}
$$

Let

$$
\lambda_{1}=\frac{\beta_{H} I_{V}}{P_{H}}>0
$$

and

$$
\lambda_{2}=\frac{\beta_{H} I_{F F}}{P_{H}}>0
$$

Substituting Equation (5) and Equation (6) into Equation (4), one can obtain Equation (7)

$$
\frac{d S_{H}}{d t} \geq \mu_{H} P_{H}+\theta R_{H}-\left(\lambda_{1}+\lambda_{2}+\mu_{H}\right) S_{H}
$$

Equation (7) can further be expressed as Equation (8)

$$
\frac{d S_{H}}{d t}+\left(\lambda_{1}+\lambda_{2}+\mu_{H}\right) S_{H} \geq \mu_{H} P_{H}+\theta R_{H}
$$

Seeking for an integrating factor $(I F)$

$$
I F=\exp \left[\int_{0}^{t}\left(\lambda_{1}+\lambda_{2}+\mu_{H}\right) d t\right]
$$

Hence Equation (9) can therefore be simplified as Equation (10)

$$
I F=\exp \left\{\int_{0}^{t}\left[\lambda_{1}(s)+\lambda_{2}(s)\right] d s+\left(\mu_{H}\right) t\right\}
$$

Multiplying through Equation (10) by Equation (8) gives Equation (11)

$$
\begin{aligned}
& \exp \left\{\int_{0}^{t}\left[\lambda_{1}(s)+\lambda_{2}(s)\right] d s+\left(\mu_{H}\right) t\right\} \frac{d S_{H}}{d t}+ \\
& \left.\exp \left\{\int_{0}^{t}\left[\lambda_{1}(s)+\lambda_{2}(s)\right] d s+\left(\mu_{H}\right) t\right\}\left(\lambda_{1}+\lambda_{2}+\mu_{H}\right) S_{H}\right\} \\
& \geq \exp \left\{\int_{0}^{t}\left[\lambda_{1}(s)+\lambda_{2}(s)\right] d s+\left(\mu_{H}\right) t\right\}\left(\mu_{H} P_{H}+\theta R_{H}\right)
\end{aligned}
$$

Equation (12) is obtained by simplifying Equation (11)

$$
\left.\begin{array}{l}
\frac{d}{d t}\left[S_{H}(t) \exp \left\{\int_{0}^{t}\left[\lambda_{1}(s)+\lambda_{2}(s)\right] d s+\left(\mu_{H}\right) t\right\}\right] \\
\geq\left(\mu_{H} P_{H}+\theta R_{H}\right) \exp \left\{\int_{0}^{t}\left[\lambda_{1}(s)+\lambda_{2}(s)\right] d s+\left(\mu_{H}\right) t\right\}
\end{array}\right\}
$$

Equation (13) is also obtained by Multiplying Equation (12) by $d t$

$$
\begin{aligned}
& d\left[S_{H}(t) \exp \left\{\int_{0}^{t}\left[\lambda_{1}(s)+\lambda_{2}(s)\right] d s+\left(\mu_{H}\right) t\right\}\right] \\
& \geq\left[\left(\mu_{H} P_{H}+\theta R_{H}\right) \exp \left\{\int_{0}^{t}\left[\lambda_{1}(s)+\lambda_{2}(s)\right] d s+\left(\mu_{H}\right) t\right\}\right] d t
\end{aligned}
$$

Integrating both sides of Equation (13), one obtains equation (14)

$$
\left.\begin{array}{l}
S_{H}(\hat{t}) \exp \left\{\int_{0}^{\hat{t}}\left[\lambda_{1}(s)+\lambda_{2}(s)\right] d s+\left(\mu_{H}\right) \hat{t}\right\} \\
\geq \int_{0}^{\hat{t}}\left[\left(\mu_{H} P_{H}+\theta R_{H}\right) \exp \left\{\int_{0}^{\hat{t}}\left[\lambda_{1}(z)+\lambda_{2}(z)\right] d z+\left(\mu_{H}\right) \hat{t}\right\}\right] d \hat{t}+S(0)
\end{array}\right\}
$$


Dividing Equation (3.18) by $\exp \left\{\int_{0}^{\hat{t}}\left[\lambda_{1}(s)+\lambda_{2}(s)\right] d s+\left(\mu_{H}\right) \hat{t}\right\}$ and simplifying further, Equation (14) then becomes Equation (15)

$$
\begin{aligned}
& S_{H}(\hat{t}) \geq S(0) \exp \left[-\left\{\int_{0}^{\hat{t}}\left[\lambda_{1}(s)+\lambda_{2}(s)\right] d s+\left(\mu_{H}\right) \hat{t}\right\}\right]+ \\
& \exp \left[-\left\{\int_{0}^{\hat{t}}\left[\lambda_{1}(s)+\lambda_{2}(s)\right] d s+\left(\mu_{H}\right) \hat{t}\right\}\right] \times \\
& \left.\int_{0}^{\hat{t}}\left[\left(\mu_{H} P_{H}+\theta R_{H}\right) \exp \left\{\int_{0}^{\hat{t}}\left[\lambda_{1}(z)+\lambda_{2}(z)\right] d z+\left(\mu_{H}\right) \hat{t}\right\}\right] d \hat{t}\right\}
\end{aligned}
$$

The right-hand side of Equation (15) is positive, which can be expressed as Equation (16)

$$
\begin{aligned}
& S(0) \exp \left[-\left\{\int_{0}^{\hat{t}}\left[\lambda_{1}(s)+\lambda_{2}(s)\right] d s+\left(\mu_{H}\right) \hat{t}\right\}\right]+ \\
& \exp \left[-\left\{\int_{0}^{\hat{t}}\left[\lambda_{1}(s)+\lambda_{2}(s)\right] d s+\left(\mu_{H}\right) \hat{t}\right\}\right] \times \\
& \left.\int_{0}^{\hat{t}}\left[\left(\mu_{H} P_{H}+\theta R_{H}\right) \exp \left\{\int_{0}^{\hat{t}}\left[\lambda_{1}(z)+\lambda_{2}(z)\right] d z+\left(\mu_{H}\right) \hat{t}\right\}\right] d \hat{t}\right\}
\end{aligned}
$$

From Equation (16), it can be concluded that $S_{H}(\hat{t})$ is positive and it is given by Equation (17) below

$$
S_{H}(\hat{t})>0
$$

Similarly, it can be shown that $I_{H}(0)>0, R_{H}(0)>0$, $S_{V}(0)>0, I_{V}(0)>0, \quad S_{F F}(0)>0, \quad I_{F F}(0)>0 \quad$ and

$$
\begin{aligned}
& D_{H}=\left\{\left(S_{H}, I_{H}, R_{H}\right) \in \mathbb{R}_{+}^{3}: S_{H}+I_{H}+R_{H} \leq \frac{P_{H}}{\mu_{H}}\right\} \\
& D_{V}=\left\{\left(S_{V}, I_{V}\right) \in \mathbb{R}_{+}^{2}: S_{V}+I_{V} \leq \frac{P_{V}}{\mu_{V}}\right\} \\
& D_{F F}=\left\{\left(S_{F F}, I_{F F}\right) \in \mathbb{R}_{+}^{2}: S_{F F}+I_{F F} \leq \frac{P_{F F}}{\mu_{F F}}\right\} \\
& D_{U_{M}}=0<U_{M}<\frac{P_{F F}}{\mu_{F F}} \\
& \Omega=\left\{\left(S_{H}, I_{H}, R_{H}, S_{V}, I_{V}, S_{F F}, I_{F F}, U_{M}\right) \in \mathbb{R}_{+}^{8} \mid S_{H}+I_{H}+R_{H}=P_{H}\right\}
\end{aligned}
$$

where $P_{H}$ is constant.

The above model (3.6) can be solved by equating the right-hand side of the model to zero. This computation shows that the model always has a disease free equilibrium (DFE) at $E_{0}=\left(S_{H}^{0}, 0,0, S_{V}^{0}, 0, S_{F F}^{0}, 0,0\right) \quad$ where, $S_{H}=P_{H}, I_{H}=0, R_{H}=0, S_{V}=P_{V}, I_{V}=0, S_{F F}=P_{F F}, I_{F F}=0$ and $U_{M}=0 \quad$ and $\quad$ a $\quad$ unique endemic equilibrium $E_{e e}=\left(S_{H}^{1}, I_{H}^{1}, R_{H}^{1}, S_{V}^{1}, I_{V}^{1}, S_{F F}^{1}, I_{F F}^{1}, U_{M}^{1}\right)$ in $\Omega$.

The basic reproduction number $\left(R_{0}\right)$ is defined as the average number of new cases of an infection caused by one typical infected individual in a population consisting of susceptible only [10].
To calculate $R_{0}$ for the above model using the Next Generation Operator approach we let $T$ be the state of the disease or the transmission state of the disease of Equation (3). 
The transmission state of Equation (3) is given by Equation (19)

$$
\left.\begin{array}{l}
T_{1}=\frac{\beta_{H} S_{H} I_{V}}{P_{V}}+\frac{\beta_{H} S_{H} I_{F F}}{P_{H}} \\
T_{2}=\frac{\beta_{V} S_{V} I_{F F}}{P_{V}}+\frac{\eta \beta_{V} S_{V} U_{M}}{K} \\
T_{3}=\frac{\beta_{F F} I_{F F} U_{M}}{K}
\end{array}\right\}
$$

Taking the Jacobian matrix of $T_{1}, T_{2}$ and $T_{3}$ with respect to $I_{H}, I_{V}, I_{F F}$ and $U_{M}$ gives, one can obtain Equation (20) below

$$
T=\left(\begin{array}{cccc}
0 & \frac{\beta_{H} S_{H}}{P_{H}} & \frac{\beta_{H} S_{H}}{P_{H}} & 0 \\
0 & 0 & \frac{\beta_{V} S_{V}}{P_{V}} & \frac{\eta \beta_{V} S_{V}}{K} \\
0 & 0 & \frac{\beta_{F F} U_{M}}{K} & \frac{\beta_{F F} I_{F F}}{K} \\
0 & 0 & 0 & 0
\end{array}\right)
$$

Evaluating $T$ at the disease-free equilibrium also gives Equation (21)

$$
T=\left(\begin{array}{cccc}
0 & \beta_{H} & \beta_{H} & 0 \\
0 & 0 & \beta_{V} & \frac{\eta \beta_{V} P_{V}}{K} \\
0 & 0 & 0 & 0 \\
0 & 0 & 0 & 0
\end{array}\right)
$$

Let $\sum$ be the transition state of Equation (3).

Hence the transition state of the disease can be expressed as Equation (22)

$$
\left.\begin{array}{l}
V_{1}=-\left(\gamma+\mu_{H}\right) \\
V_{2}=-\mu_{V} I_{V} \\
V_{3}=-\mu_{F F} I_{F F} \\
V_{4}=\sigma_{V} I_{V}+\sigma_{F F} I_{F F}-\mu_{E} U_{M}
\end{array}\right\}
$$

Taking the Jacobian matrix of $V_{1}, V_{2}, V_{3}$, and $V_{4}$ with respect to $I_{H}, I_{V}, I_{F F}$ and $U_{M}$ gives

$$
\Sigma=\left(\begin{array}{cccc}
-\left(\gamma+\mu_{H}\right) & 0 & 0 & 0 \\
0 & -\mu_{V} & 0 & 0 \\
0 & 0 & -\mu_{F F} & 0 \\
0 & \sigma_{V} & \sigma_{F F} & -\mu_{E}
\end{array}\right)
$$

From Equation (23), we have Equation (24)

$$
-\Sigma=\left(\begin{array}{cccc}
\left(\gamma+\mu_{H}\right) & 0 & 0 & 0 \\
0 & \mu_{V} & 0 & 0 \\
0 & 0 & \mu_{F F} & 0 \\
0 & -\sigma_{V} & -\sigma_{F F} & \mu_{E}
\end{array}\right)
$$

Hence the inverse of Equation (24) is computed as Equation (25)

$$
-\Sigma^{-1}=\left(\begin{array}{cccc}
\frac{1}{\left(\gamma+\mu_{H}\right)} & 0 & 0 & 0 \\
0 & \frac{1}{\mu_{V}} & 0 & 0 \\
0 & 0 & \frac{1}{\mu_{F F}} & 0 \\
0 & \frac{\sigma_{V}}{\mu_{V} \mu_{E}} & \frac{\sigma_{F F}}{\mu_{F F} \mu_{E}} & \frac{1}{\mu_{E}}
\end{array}\right)
$$

Let $K_{L}=$ Next Generation Matrix (NGM) with large domain. Therefore, the NGM is computed as Equation (26)

$$
K_{L}=-T \Sigma^{-1}
$$

hence

$$
K_{L}=\left(\begin{array}{cccc}
0 & \beta_{H} & \beta_{H} & 0 \\
0 & 0 & \beta_{V} & \frac{\eta \beta_{V} P_{V}}{K} \\
0 & 0 & 0 & 0 \\
0 & 0 & 0 & 0
\end{array}\right)\left(\begin{array}{cccc}
\frac{1}{\left(\gamma+\mu_{H}\right)} & 0 & 0 & 0 \\
0 & \frac{1}{\mu_{V}} & 0 & 0 \\
0 & 0 & \frac{1}{\mu_{F F}} & 0 \\
0 & \frac{\sigma_{V}}{\mu_{V} \mu_{E}} & \frac{\sigma_{F F}}{\mu_{F F} \mu_{E}} & \frac{1}{\mu_{E}}
\end{array}\right)
$$

Simplifying Equation (27) above gives Equation (28) below

$$
K_{L}=\left(\begin{array}{cccc}
0 & \frac{\beta_{H}}{\mu_{H}} & \frac{\beta_{H}}{\mu_{F F}} & 0 \\
0 & \frac{\eta \beta_{V} P_{V} \sigma_{V}}{K \mu_{V} \mu_{E}} & \frac{\beta_{V}}{\mu_{V V}}+\frac{\eta \beta_{V} P_{V} \sigma_{F F}}{K \mu_{F F} \mu_{E}} & \frac{\eta \beta_{V} P_{V}}{K \mu_{E}} \\
0 & 0 & 0 & 0 \\
0 & 0 & 0 & 0
\end{array}\right)
$$

Let $\lambda_{n}$ be the eigenvalues of $K_{L}$. Then the eigenvalues of Equation (28) is computed as Equation (29)

$$
\operatorname{det}\left(K_{L}-\lambda I\right)=0
$$

Where $I$ is a $4 \times 4$ identity matrix and $n=1,2,3,4$.

Hence the eigenvalues of Equation (28) are obtained as Equation (30) 


$$
\left.\begin{array}{l}
\lambda_{1}=0 \\
\lambda_{2}=0 \\
\lambda_{3}=0 \\
\lambda_{4}=\frac{\eta \beta_{V} P_{V} \sigma_{V}}{K \mu_{V} \mu_{E}}
\end{array}\right\}
$$

The basic reproductive number $\left(R_{0}\right)$ is the spectral radius of $K_{L}$ which is written as Equation (31)

$$
R_{0}=\rho\left(K_{L}\right)
$$

Hence the basic reproduction number is largest eigenvalue of Equation (30).

Therefore

$$
R_{0}=\lambda_{4}
$$

Hence the basic reproduction number is given by Equation (33)

$$
J_{E_{0}}=\left(\begin{array}{cccccccc}
-\mu_{H} & 0 & \theta & 0 & -\beta_{H} & 0 & -\beta_{H} & 0 \\
0 & -\left(\gamma+\mu_{H}\right) & 0 & 0 & \beta_{H} & 0 & \beta_{H} & 0 \\
0 & \gamma & -\left(\theta+\mu_{H}\right) & 0 & 0 & 0 & 0 & 0 \\
0 & 0 & 0 & -\mu_{V} & 0 & 0 & -\beta_{V} & -\frac{\eta \beta_{V} P_{V}}{K} \\
0 & 0 & 0 & 0 & -\mu_{V} & 0 & \beta_{V} & \frac{\eta \beta_{V} P_{V}}{K} \\
0 & 0 & 0 & 0 & 0 & -\mu_{F F} & 0 & 0 \\
0 & 0 & 0 & 0 & 0 & 0 & -\mu_{F F} & 0 \\
0 & 0 & 0 & 0 & \sigma_{V} & 0 & \sigma_{F F} & -\mu_{E}
\end{array}\right)
$$

Let $\lambda_{i}$ be the eigenvalues of $J_{E_{0}}$, for $i=1,2,3,4,5,6,7,8$, the eigenvalues is computed as

$$
\left.\begin{array}{l}
\lambda_{1}=-\mu_{H}<0 \\
\lambda_{2}=-\left(\gamma+\mu_{H}\right)<0 \\
\lambda_{3}=-\left(\theta+\mu_{H}\right)<0 \\
\lambda_{4}=-\mu_{V}<0 \\
\lambda_{5}=-\mu_{F F}<0 \\
\lambda_{6}=-\mu_{F F}<0 \\
\lambda_{7}=-\frac{1}{2}\left(\mu_{V}+\mu_{E}-\sqrt{4 \mu_{V} \mu_{E} R_{0}+\mu_{V}^{2}-2 \mu_{V} \mu_{E}+\mu_{E}^{2}}\right)<0 \\
\lambda_{8}=-\frac{1}{2}\left(\mu_{V}+\mu_{E}+\sqrt{4 \mu_{V} \mu_{E} R_{0}+\mu_{V}^{2}-2 \mu_{V} \mu_{E}+\mu_{E}^{2}}\right)<0
\end{array}\right\}
$$

From Equation (35), to show that $\lambda_{7}$ negative, we impose a negativity condition to it.

$\lambda_{7}$ is negative if and only if Equation (36) below holds

$$
\sqrt{4 \mu_{V} \mu_{E} R_{0}+\mu_{V}^{2}-2 \mu_{V} \mu_{E}+\mu_{E}^{2}}<\left(\mu_{V}+\mu_{E}\right)
$$

Simplifying Equation (36), $R_{0}$ is obtained as Equation (37) below

$$
R_{0}<1
$$

From Equation (37), it implies that $\lambda_{7}$ is negative.

Since the eigenvalues of $J_{E_{0}}$ are all negatives it therefore shows that the disease-free equilibrium is locally asymptotically stable for $R_{0}<1$. 


\subsection{The Stability of the Endemic Equilibrium}

The endemic equilibrium $E_{e e}$ of the model is locally asymptotically stable if and only if $R_{0}>1$. However it is very difficult to deal with the stability of the endemic equilibrium analytically due to the nature of transmission model (i.e.
Model 3). By numerical approach, the endemic equilibrium is locally asymptotically stable. Different initial conditions were applied for the simulation. Those obits shown to be the same point as time evolve.

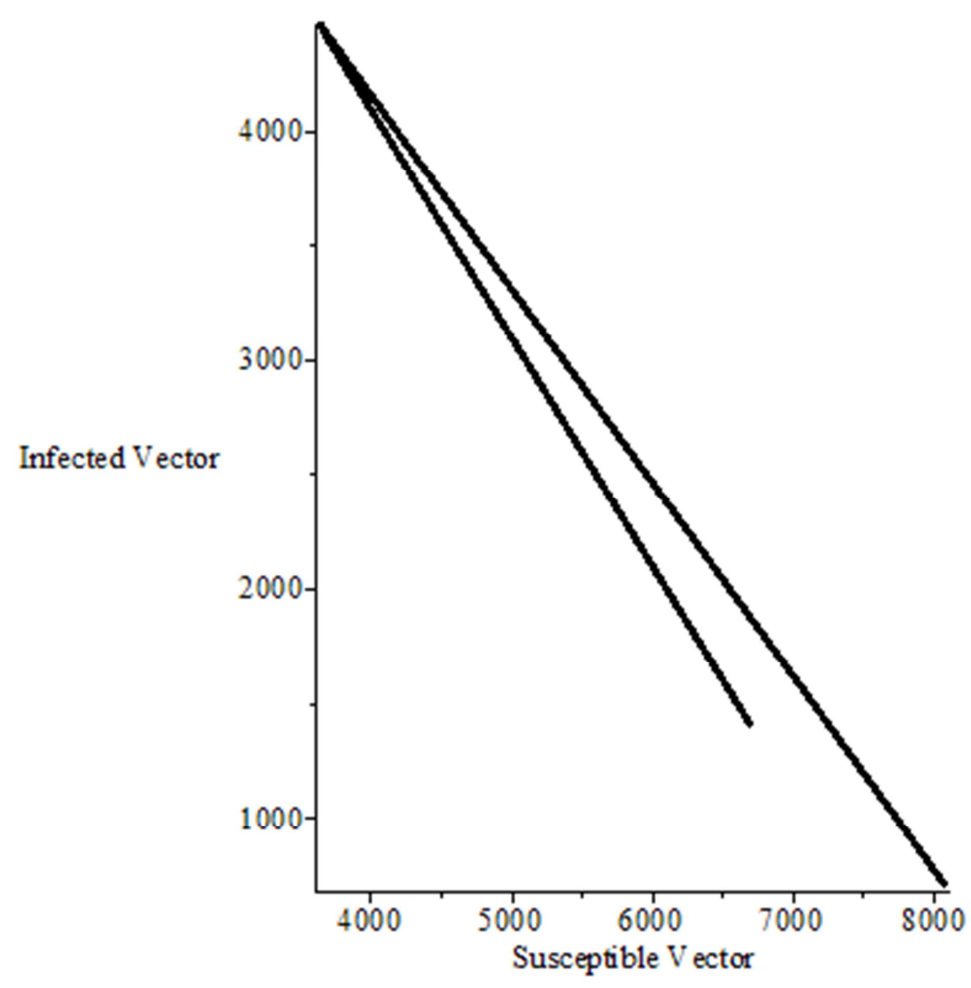

Figure 5. Phase portrait of Model 3 in $S_{V}-I_{V}$ plane.

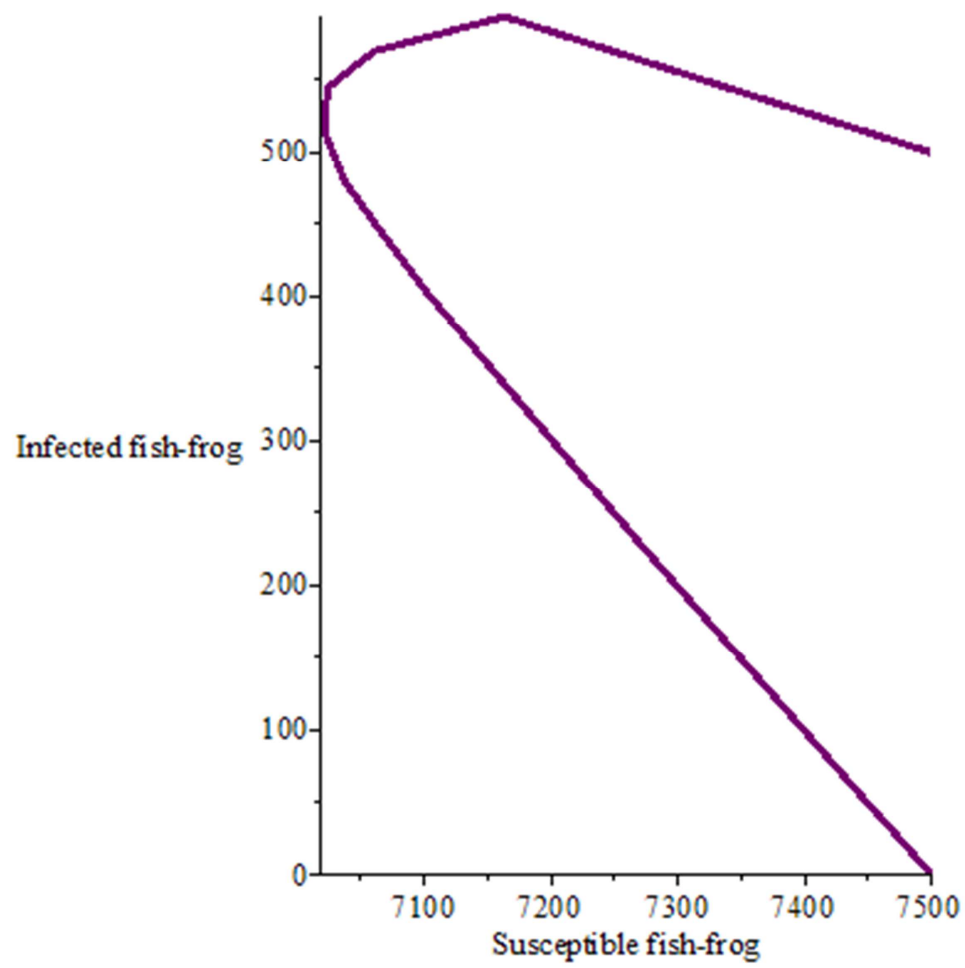

Figure 6. Phase portrait of Model 3 in $S_{F F}-I_{F F}$ plane . 
Table 3. Description of parameters used in Equation (3).

\begin{tabular}{|c|c|}
\hline Symbol & Description \\
\hline$\beta_{H}$ & The effective contact rate between the vector, fish-frog and susceptible human \\
\hline$\beta_{V}$ & The effective contact rate between fish-frog and susceptible vector \\
\hline$\beta_{F F}$ & The effective contact rate between the susceptible fish-frog and Mycobacterium Ulcerans \\
\hline$\gamma$ & The recovery rate of infected humans \\
\hline$\theta$ & The rate of loss of immunity of recovered humans \\
\hline$\mu_{H}$ & Birth rate of the human population \\
\hline$\mu_{V}$ & Birth rate of the vector population \\
\hline$\mu_{F F}$ & Birth rate of the fish-frog population \\
\hline$r V$ & The growth rate of the vector population \\
\hline$r F$ & The growth rate of the fish-frog population \\
\hline$K$ & The environmental carrying capacity of the bacteria population \\
\hline$\sigma_{F F}$ & $\begin{array}{l}\text { Rate of shedding of Mycob } \\
\text { acterium Ulcerans into the environment by fish-frog }\end{array}$ \\
\hline$\sigma_{V}$ & Rate of shedding of Mycobacterium Ulcerans into the environment by the vector \\
\hline$\mu_{E}$ & Rate at which Mycobacterium Ulcerans are cleared from the environment. \\
\hline
\end{tabular}

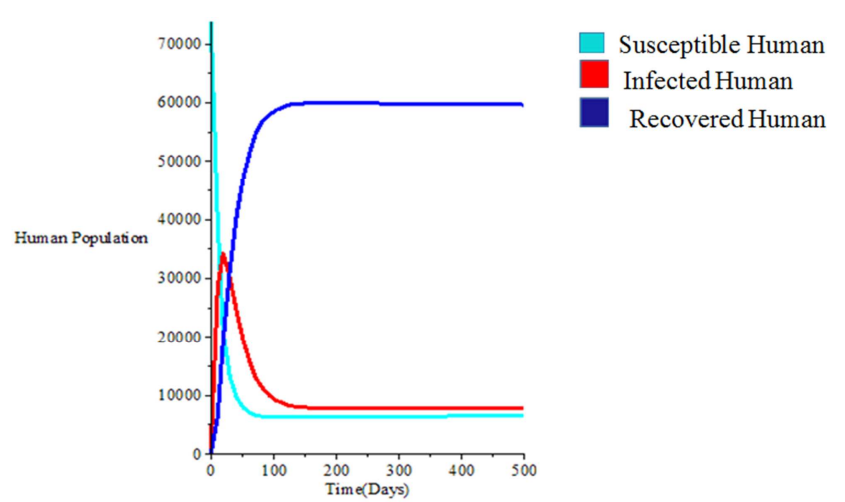

Figure 7. Dynamics of Human compartment of Equation (3).

\section{Results and Discussion}

Numerical simulation of the Equation (3.6) was carried out using Maple inbuilt functions. Analysis of the response of model parameters on the transmission dynamics of the disease was studied and the evaluation of the basic reproduction number was made. Since, most of the parametric values are not readily available it is needed to assign some arbitrary values. However, some are available at $[6,11,12]$. The initial conditions were taken at initial time of zero (0) and the final time was considered as 500, 500, 500, 500, 520, 2000, 2000, 2000, 7000, 8000 and finally 10000 . The results of the simulation study are presented in Figure 8.

From Figure 7 the model simulations show that the susceptible human population decrease and becomes steady with time. The infected human population also increases so sharply and becomes asymptotic with time. This is due to the rate at which a susceptible human is bitten by an infected water bug and also the rate at which a susceptible human feed on an infected fish-frog. It can be observed that the Recovered human population increases to a certain point and it then decreases asymptotically with time.

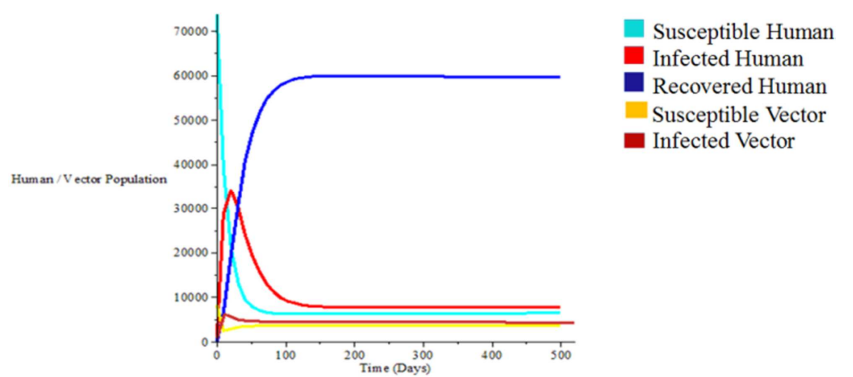

Figure 8. Dynamics of the Human and the Vector compartments.

Figure 8 shows the general interactions between human and vector population in the study areas with time. It's also shows that there will be a widespread occurrence of the Buruli Ulcer disease in study areas.

The basic reproduction number was calculated as

$$
R_{0}=1.207710000
$$

Equation Chapter (Next) Section 1 (38)

The positivity of solution to the model was proved as shown in Equation (4) to (17). The Basic Reproduction Number $\left(R_{0}\right)$ was computed from the deterministic model that was developed in Equation (4). The model's equilibria were determined and conditions for the equilibria were also established, and their stabilities were investigated in terms of the classic threshold $\left(R_{0}\right)$. It is well known in disease transmission modelling that, a classical necessary condition for minimizing or eliminating disease completely is that the basic reproduction number $\left(R_{0}\right)$, must be less than unity. If the basic reproduction number is greater than unity, then the disease will eventually spread in a population. The diseasefree equilibrium (DFE) is found to be locally asymptotically stable for $R_{0}<1$ as it's shown in Equation (37). The endemic equilibrium is also found to be locally asymptotically stable for $R_{0}>1$.

A simulation was performed on the model using some of the parameters in Table 3. The results obtained indicate that, 
the disease has attained a steady state, this is typically shown in Figure 8. In examining the state of Buruli Ulcer in the study area, since $R_{0}>1$ it proves that the number of infected persons in the communities will increase with time.

\section{Conclusions}

The study developed a deterministic model for Buruli Ulcer based on the transmission mechanism of the disease in the study area. The basic reproduction number $R_{0}$ derived was found to be greater than unity (i.e. $R_{0}>1$ ), this shows that, the disease will spread horizontally across the population, and the estimated value of $R_{0}$ was 1.20771 . The transmission dynamics of Buruli Ulcer model of the Susceptible, Infected and Recovered (SIR) type showed that, the disease will continue to spread at the study areas as long as the reservoir for Mycobacterium Ulcerans continue to increase and also as long as enough infected water bugs and infected fish or infected frog continue to increase. However, the spread of the disease (Buruli Ulcer) will be minimised when control measures are being implemented. It is therefore recommended that People in the affected communities should be given adequate education on the Buruli Ulcer disease, how contaminated water or stagnant water play a major role in the transmission of the disease and how the disease should be treated properly to minimize its spread.

\section{Acknowledgements}

The authors express their appreciation to Prof. Richard Amankwah of the University of Mines and Technology, Tarkwa for his assistance towards the publication of this paper.

\section{References}

[1] Daley, D. J. and Gani, J. (2005), Epidemic Modelling and Introduction, NY: Cambridge University Press.

[2] Moghadas, S. M. (2017), Gaining insights into human viral diseases through mathematics European Journal of Epidemiology, Vol 21, pp. 337-342.
[3] Effah, E., Nyame F, and Amankwah, R. K, (2016), "Spatial Distribution of Buruli Ulcer (BU) in Communities along River Offin in Central Ghana", $4^{\text {th }}$ UMaT Biennial International Mining and Mineral Conference.

[4] Clancey, J., Dodge, R. and Lunn, H. F., (1962), Study of a mycobacterium causing skin ulceration in Uganda, Ann Soc Belg Med Trop, 4, pp. 585-590.

[5] Asiedu, K., Raviglione, M. C., Scherpbier, R. (2016), "World Health Organization and Global Buruli Ulcer Initiative", Buruli ulcer: Mycobacterium ulcerans infection (No. WHO/CDS/CPE/GBUI/2000.1). Geneva: World Health Organization.

[6] Scott, B., Curtis, V., Rabie, T. and Garbrah-Aidoo, N., (2007), Health in our hands, but not in our heads: understanding hygiene motivation in Ghana, Health Policy and planning, 22, (4), pp. 225-233.

[7] Nyabadza, F. and Bonyah, E. (2015), "On the Transmission Dynamics of Buruli ulcer in Ghana: Insights through a mathematical model", BMC research notes, pp. 656.

[8] Merritt, R. W., Walker, E. D., Small, P. L., Wallace, J. R., Johnson, P. D., Benbow, M. E. and Boakye, D. A. (2010), "Ecology and Transmission of Buruli Ulcer Disease: a systematic review", PLoS neglected tropical diseases, Vol. 4, No. 12, pp. 911.

[9] Eddyani, M., Ofori-Adjei, D., Teugels, G., De Weirdt, D., Boakye, D., Meyers, W. M. and Portaels, F., (2018), Potential role for fish in transmission of Mycobacterium ulcerans disease (Buruli ulcer): an environmental study. Appl. Environ. Microbiol., 70, (9), pp. 5679-5681.

[10] Diekmann, O., Heesterbeek, J. A. P. and Roberts, M. G., (2009), "The Construction of Next Generation Matrices for Compartmental Epidemic Models", Journal of the Royal Society Interface, Vol. 7, No. 47, pp. 873-885.

[11] Dontwi, I. K., Obeng-Denteh, W., Andam, E. A. and ObiriApraku, L., (2014), A mathematical model to predict the prevalence and transmission dynamics of tuberculosis in Amansie West District, Ghana, Journal of Advances in Mathematics and Computer Science, pp. 402-425.

[12] Bonyah, E., (2015), Mathematical models for the study of buruli ulcer dynamics in Ghana (Doctoral dissertation). 